\title{
COMPROMETIMENTO ORGANIZACIONAL: QUESTÕES QUE CERCAM SUA NATUREZA E OS SEUS LIMITES CONCEITUAIS
}

ORGANIZATIONAL COMMITMENT: SOME ISSUES SURROUNDING ITS NATURE AND CONCEPTUAL BOUNDS

COMPROMISO ORGANIZACIONAL: CUESTIONES RELATIVAS A SU NATURALEZA Y SUS
LÍMITES CONCEPTUALES

Igor Gomes Menezes*

Carolina Villa Nova Aguiar ${ }^{*}$

Antonio Virgilio Bittencourt Bastos ${ }^{* *}$

\begin{abstract}
RESUMO
O comprometimento organizacional, um dos fenômenos mais investigados no campo do comportamento organizacional, pode ser considerado um construto polissêmico e multifacetado. Este trabalho buscou discutir a complexidade e dispersão que cerca o conceito de comprometimento organizacional, envolvendo três problemas centrais: 10) a quantidade de interfaces teóricas entre comprometimento organizacional e outros construtos da área organizacional; 2o) o grande desequilíbrio entre estudos que tomam o construto como uma atitude em detrimento de definições que enfatizem indicadores comportamentais; e $3^{\circ}$ ) a dimensionalidade do construto e a falta de consenso a respeito de quantas e quais são as bases constitutivas do comprometimento organizacional. Além de sistematizar os principais problemas sobre o construto, este estudo sugere alguns caminhos de investigação teórica e empírica que poderiam minimizar os problemas que cercam esta vertente de pesquisa, em razão das questóes conceituais que caracterizam o seu construto central.
\end{abstract}

Palavras-chave: Comprometimento organizacional. Polissemia do comprometimento. Dimensionalidade do comprometimento. Perspectivas teóricas.

\footnotetext{
ABSTRACT

Organizational commitment, although one of the most investigated phenomena in the field of organizational behavior, may still be considered as a multifaceted and polysemous construct. This work aims to present the

Texto recebido em 15 de novembro de 2013 e aprovado para publicação em 17 de novembro de 2015 .

Doutor em Psicologia. Universidade Federal da Bahia (UFBA). E-mail: igor.menezes@ufba.br.

* Doutora em Psicologia. UFBA. E-mail: carol.vna@gmail.com.

${ }^{* * *}$ Doutor em Psicologia. UFBA. Endereço: Rua Coronel José Galdino de Souza, 225, ap. 104 - Federação, Salvador-BA, Brasil. CEP: 41950-150. E-mail: antoniovirgiliobastos@gmail.com.
} 
complexity and dispersion that revolves around the concept of organizational commitment into three central problems: 1) large number of theoretical interfaces between organizational commitment and other organizationalrelated constructs, 2) the preponderance of studies that investigate commitment as an attitude rather than researches that emphasize behavioral indicators, and 3) the construct dimensionality, and the lack of a consensus about how many and which bases constitutes organizational commitment. In addition to discuss these main issues on the topic, this work suggests some theoretical and empirical research ways that could minimize some problems surrounding the mainstream organizational commitment literature, based on conceptual questions that revolve around this central construct.

Keywords: Organizational commitment. Commitment polysemy. Commitment dimensionality. Theoretical approaches.

\section{RESUMEN}

El compromiso organizacional, uno de los fenómenos más investigados en el campo del comportamiento organizacional, puede ser considerado un constructo polisémico y multifacético. El presente estudio tuvo como objetivo discutir la complejidad y dispersión en torno del concepto de compromiso organizacional, involucrando a tres problemas fundamentales: 1) la cantidad de conexiones teóricas entre el compromiso organizacional y otros constructos del área organizacional, 2) el gran desequilibrio ente estudios que asumen el constructo como una actitud, en detrimento de definiciones que enfaticen indicadores de comportamiento, 3) la dimensionalidad del constructo y la falta de consenso sobre cuántas y cuáles son las bases constitutivas del compromiso organizacional. Además de sistematizar los principales problemas sobre el constructo, este estudio sugiere algunos caminos de investigación teórica y empírica que podrían minimizar los problemas relacionados con esta línea de investigación, en función de las cuestiones conceptuales que caracterizan su constructo central.

Palabras clave: Compromiso organizacional. Polisemia del compromiso. Dimensionalidad del compromiso. Enfoques teóricos.

\section{INTRODUÇÃO}

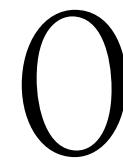
comprometimento organizacional é um dos construtos mais investigados dentro do campo do comportamento organizacional, devido à centralidade que a sua noção desempenha na constituição e funcionamento de unidades sociais, como equipes e organizações. $\mathrm{Na}$ ampla gama de estudos sobre o tema, o conceito tem sido trabalhado com base em significados nem sempre 
convergentes. Essa pluralidade de acepções tem tornado, ao longo de décadas de investigação, o comprometimento organizacional um construto cada vez mais polissêmico, complexo e multifacetado.

De acordo com Osigweh (1989), os conceitos, por serem classificações saturadas de significados, constituem os blocos de construção da ciência e, quando são inadequadamente estruturados, podem afetar negativamente todo o empreendimento científico. Conceitos mal especificados geram proposiçôes de pesquisa ambíguas e dificultam a produção cumulativa de conhecimento (Achinstein, 1968; Bagozzi \& Fornell, 1982). Embora se reconheça a necessidade de um elevado grau de conhecimento e sistematização do fenômeno para o desenvolvimento eficiente de uma teoria, é notório que as diferentes perspectivas teóricas sobre comprometimento com a organização ainda não acederam à elaboração de um conceito de comprometimento organizacional mais acurado e bem delimitado. Tendo em vista a pluralidade de conceitos voltados a descrever o comprometimento organizacional, este artigo tem por objetivo apresentar, analisar e discutir a dispersão que se constata nos estudos teóricos e empíricos sobre comprometimento organizacional.

Basicamente são três os problemas centrais que dificultam a elaboração de um conceito mais claramente delimitado e conciso sobre o comprometimento organizacional. O primeiro deles diz respeito aos limites imprecisos entre comprometimento organizacional e construtos correlatos. $\mathrm{O}$ segundo problema relaciona-se ao surgimento de diferentes perspectivas teóricas para a investigação do fenômeno, com destaque para as abordagens atitudinal e comportamental. E o terceiro está associado à pluralidade das bases que diferenciam a natureza do vínculo estabelecido entre trabalhador e organização, conhecido também como o problema da dimensionalidade do construto.

\section{OS LIMITES CONCEITUAIS ENTRE COMPROMETIMENTO ORGANIZACIONAL E OUTROS CONSTRUTOS}

É difícil precisar ao certo o primeiro conceito que se tornou central para a investigação do comprometimento organizacional. Entretanto uma das primeiras concepçóes sobre o construto encontra-se associada à perspectiva sociológica e foi desenvolvida por Becker (1960), no âmbito da teoria de side-bets. Segundo o autor, o comprometimento diz respeito ao "engajamento em linhas consistentes de ação" (Becker, 1960, p. 33) e envolve três elementos centrais: a persistência do comportamento durante um período significativo; a rejeição de alternativas de trabalho; e a perseguição de uma meta, não obstante a adversidade externa. Para Becker, um indivíduo permanece vinculado a uma organização devido 
aos custos e benefícios associados à sua saída, além da necessidade de ajustarse individualmente a posiçôes sociais. Os side-bets correspondem, portanto, às trocas laterais entre indivíduo e organização, que fazem com que um trabalhador busque comportar-se de determinada maneira devido aos custos associados à sua saída.

Ao trazer a ideia de que os indivíduos se engajam em linhas consistentes de ação que favorecem o prolongamento do vínculo com sua organização, Becker foi o primeiro autor a aproximar o conceito de comprometimento organizacional da noção de permanência. Tal noção influenciou o desenvolvimento da medida de comprometimento organizacional que teve larga utilização na década de 1980, o organizational commitment questionnaire (OCQ). Com a criação do OCQ, Mowday, Steers, e Porter (1979) preconizaram que o comprometimento organizacional deveria ser avaliado com base em duas dimensões: identificação e intençôes de permanência. Entretanto, ainda que o desenvolvimento de itens para avaliar a noção de permanência na organização estivessem atrelado à constituição da estrutura do comprometimento como construto, os itens que compunham a dimensão de permanência não demonstraram correlação com os demais itens da dimensão afetiva (identificação), formando uma estrutura fatorial diferenciada. Os estudos de Mowday et al. (1979) trouxeram as primeiras evidências empíricas que sugeriram que a permanência apresentava elementos conceitualmente distintos da noção de comprometimento, presente nos demais itens da escala.

Embora, historicamente, a noção de permanência tenha ocupado lugares distintos, ora como parte do construto, ora como consequente, tal relação entre comprometimento e permanência contribuiu com o desenvolvimento de uma perspectiva instrumental para o comprometimento organizacional (Etzioni, 1961; McGee \& Ford, 1987; Meyer \& Allen, 1984). Nessas pesquisas, a permanência na organização associou-se diretamente à necessidade de manutenção do vínculo, quer seja por razões pessoais, quer por motivos profissionais, como investimentos realizados, remuneração, benefícios, entre outros. Entretanto, atualmente, crescem os estudos que seguem a tendência de Mowday et al. (1979), os quais defendem a ideia de que comprometimento organizacional e desejo de permanência na organização são fenômenos distintos (Bastos \& Aguiar, 2015; Cooper-Hakim \& Viswesvaran, 2005; Klein, Molloy \& Cooper, 2012; Mathieu \& Zajac, 1990; Menezes, 2006; Meyer, Stanley, Herscovitch \& Topolnytsky, 2002; Rodrigues \& Bastos, 2010; Solinger, Olffen \& Roe, 2008).

Além da associação com a permanência, o comprometimento organizacional tem sido historicamente vinculado a diferentes processos de influência social que guardam com ele correlatos teóricos. A falta de clareza conceitual sobre o 
comprometimento organizacional encontra-se embutida em uma das definições mais clássicas sobre o construto, em que Mowday et al. (1979) caracterizaram o comprometimento organizacional como "a força relativa da identificação de um indivíduo e envolvimento com uma organização particular” (p. 226), associando a ideia de comprometimento a essas duas noções. Segundo Morrow (1983), essa aproximação conceitual ocorre pelo fato de que

O crescimento de conceitos relacionados a comprometimento não tem sido acompanhado de uma segmentação cuidadosa do domínio teórico do comprometimento em termos do significado pretendido de cada conceito ou das relações entre esses conceitos (p. 486).

Morrow (1983), ao caracterizar a proximidade entre comprometimento e envolvimento, descreve que o primeiro é um atributo mais estável e fixo, enquanto o último é representado por elementos mais mutáveis e passíveis de manipulação, já que é influenciado por condiçôes relativas a uma situação específica. De um modo geral, o termo envolvimento tem sido usado na literatura organizacional para descrever o vínculo do trabalhador tanto com sua organização quanto com seu trabalho.

Em um clássico trabalho nessa área, Etzioni (1961) empregou o conceito de envolvimento para tratar do vínculo estabelecido pelo trabalhador com organizações de diferentes naturezas. $\mathrm{O}$ autor propôs três diferentes tipos de envolvimento: moral, calculativo e alienativo. Indivíduos moralmente envolvidos aceitam e se identificam com as metas da organização. Há um envolvimento calculativo, quando o vínculo se dá pelas trocas estabelecidas com a organização. Já o envolvimento alienativo refere-se à permanência na organização devido à falta de alternativas ou a um sistema comportamental de aquiescência que os força a permanecer, tal qual eles estivessem aprisionados.

A identificação organizacional é o segundo conceito que aparece incorporado à definição de comprometimento, mas que também constitui uma linha de pesquisa independente, como se comprova na meta-análise de Riketta (2005). De acordo com O'Reilly e Chatman (1986), a identificação estaria, assim, diretamente relacionada com o desejo de afiliação. Ashforth e Mael (1989) distinguem comprometimento organizacional de identificação de duas maneiras. Primeiro, o comprometimento organizacional pode refletir metas individuais que não são necessariamente úteis à coletividade; e segundo, o comprometimento é geralmente considerado como um estado afetivo, enquanto a identificação organizacional é um fenômeno perceptual ou cognitivo. Para Beyer, Hannah 
e Milton (2000), o comprometimento organizacional deve ser entendido como um construto mais amplo, sendo constituído tanto por características de envolvimento quanto por elementos de identificação.

O comprometimento organizacional tem sido associado ainda a outros construtos, como cidadania organizacional, contrato psicológico e lealdade. A dispersão conceitual do comprometimento organizacional e sua relação com outros construtos da área organizacional podem ser observadas na Figura 1.

Figura 1. Comprometimento organizacional e suas relações com outros construtos.

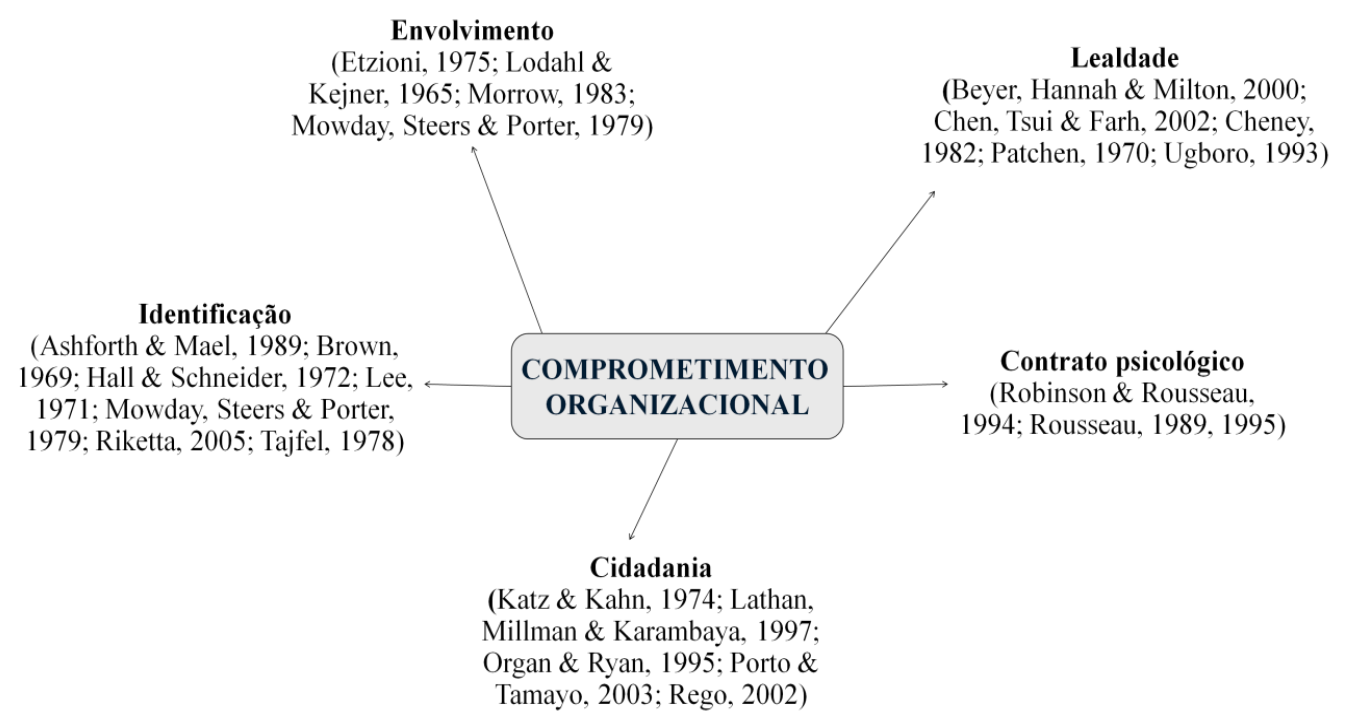

Fonte: elaborado pelos autores.

A pulverização de conceitos e a falta de limites mais claros entre comprometimento organizacional e outros construtos como os discutidos acima colocam dois principais desafios teóricos para a área de pesquisa: o primeiro, de se definir que conceitos de menor escopo devem integrar o de comprometimento (como seriam os casos de envolvimento, que abarca a dimensão afetiva; e os de identificação e lealdade, que, por vezes, tocam na dimensão cognitiva e, em outros momentos, na dimensão afetiva); e o segundo, de se verificar a possível sobreposição entre comprometimento e outros construtos também voltados para caracterizar vínculos entre indivíduo e a organização, caso em que se encontram os conceitos de contrato psicológico e de comportamentos de cidadania organizacional. 


\section{ANATUREZADOCOMPROMETIMENTOORGANIZACIONAL: UM FENÔMENO ATITUDINAL OU COMPORTAMENTAL?}

Além da falta de clareza na delimitação do fenômeno, a polissemia do comprometimento organizacional é impactada diretamente pelas diferentes abordagens teóricas que se acercam do construto. São duas as abordagens que contribuem para a diversidade de definições sobre o comprometimento: $1^{\circ}$ ) a abordagem atitudinal, que enfatiza a natureza do processo de identificação do indivíduo com objetivos e valores da organização e focaliza possíveis atitudes que predispõem futuros comportamentos de comprometimento; e $2^{\circ}$ ) a abordagem comportamental, na qual o comprometimento se expressa fundamentalmente em comportamentos ou atos, sendo as cognições elementos que fortalecem tal vínculo.

Enquanto a abordagem atitudinal examina as crenças, afetos e intenções de comportamentos que se dirigem a um objeto, sendo os comportamentos uma consequência das atitudes, a abordagem comportamental considera que as pessoas se comprometem com ações, sendo as atitudes geradas a partir de comportamentos como desempenho e proatividade. De acordo com Mowday, Porter, e Steers (1982), o comprometimento comportamental diz respeito ao processo pelo qual os indivíduos se tornam parte de uma determinada organização, assim como o modo com que eles lidam com isso. Para tal abordagem, para que se possa obter uma visão clara do comprometimento, é mais útil considerar o que os empregados fazem do que saber aquilo que relatam sobre suas opiniões e sentimentos (Benkhoff, 1997). Segundo Salancik (1977), uma pessoa comprometida não é aquela que somente relata seu desejo de permanecer no emprego, mas que, sobretudo, trabalha proativamente em defesa dos interesses da organização.

De um modo geral, ao passo que a abordagem atitudinal considera a importância das atitudes comprometidas sobre o comportamento, a abordagem comportamental enfatiza a influência de comportamentos comprometidos sobre as atitudes (Mowday, Porter \& Steers, 1982). A polarização de concepções distintas sobre a natureza do comprometimento organizacional com base nas abordagens atitudinal e comportamental tem feito com que pouca atenção esteja sendo concedida à integração entre atitudes e comportamentos de comprometimento. A maior parte das pesquisas que investigam essa relação está preocupada com a influência das atitudes sobre os comportamentos, tipicamente avaliados pela intenção de permanência na organização (Bluedorn, 1982; Marsh \& Mannari, 1977; Mobley, Griffith, Hand \& Megline, 1979; Mowday, Koberg \& McArthur, 1984; Peters, Bhagat \& O’Connor, 1981; Steers, 1977). Até mesmo estudos clássicos, como os de O’Reilly e Caldwell (1980) e de Salancik e 
Pfeffer (1978), que investigam a influência dos comportamentos nas atitudes de comprometimento, não buscam investigar os efeitos recíprocos entre tais tipos de comprometimento, sendo realizados unicamente de maneira unidirecional.

Não obstante a distinção teórica e empírica entre as abordagens atitudinal e comportamental, a literatura sobre o tema não toma essas abordagens como perspectivas concorrentes, mas complementares, reconhecendo-se a contínua necessidade de integrá-las em futuras investigaçôes. Mowday et al. (1982) destacaram a necessidade de uma inter-relação entre as perspectivas atitudinal e comportamental. De acordo com os autores, mais importante do que saber se os processos envolvidos no comprometimento começam com atitudes ou comportamentos é reconhecer que o desenvolvimento do fenômeno envolve inter-relações entre atitudes e comportamentos ao longo do tempo. Apesar da recomendação de Mowday et al. (1982), poucos estudos têm se voltado à tentativa de integração entre as abordagens do comprometimento.

\section{A DIMENSIONALIDADE DO COMPROMETIMENTO ORGANIZACIONAL}

Uma das maiores divergências entre os pesquisadores do comprometimento organizacional diz respeito à dimensionalidade do construto, que corresponde à forma como se configura sua estrutura interna; como ele se constitui qualitativamente. Mais, diz respeito à quantidade de dimensões (também conhecidos como fatores) que, geralmente, definirá o grau de complexidade de um construto. Construtos mais complexos são mais heterogêneos e tendem a requerer mais fatores para explicá-lo em toda sua amplitude.

Um dos primeiros pesquisadores da perspectiva atitudinal a suscitar o debate sobre a dimensionalidade do comprometimento organizacional foi Gouldner (1960), que identificou duas dimensóes como constituintes do construto:

a) integração, que diz respeito ao grau em que o indivíduo é ativo e se sente parte de uma determinada organização; e

b) introjeção, que corresponde ao grau em que a própria imagem de um indivíduo inclui a variedade de características e valores organizacionais aprovados. Nesse caso, a integração estaria mais ligada à noção de identificação e mais próxima, assim, de uma questão afetiva. Por outro lado, a introjeção encontra-se diretamente relacionada a um contexto normativo, em que há um compartilhamento entre valores individuais e valores organizacionais. 
Outro estudo tradicional de grande relevância para a definição do que viria a ser considerada por Allen e Meyer (1990) como uma dimensão instrumental do comprometimento organizacional foi desenvolvido por Kanter (1968), que justificou a dimensionalidade do construto com base em um modelo tridimensional:

a) comprometimento de coesão, vínculo diretamente associado às técnicas e eventos que tornam pública a imagem de um indivíduo como membro da organização e que reforçam a coesão grupal;

b) comprometimento de continuação, primeira utilização do termo continuan$c e$ (continuação), relacionada aos sacrifícios pessoais e investimentos que geram custos de deixar a organização; e

c) comprometimento de controle, vínculo que favorece a assimilação das normas da organização por parte dos seus membros que, por sua vez, moldam seus comportamentos em conformidade com essas normas.

$\mathrm{Na}$ clássica descrição de comprometimento organizacional de Mowday et al. (1979), o construto, embora concebido com base em uma perspectiva unidimensional, inclui três aspectos distintos:

a) uma forte crença e uma aceitação das metas e valores organizacionais;

b) o desejo de exercer considerável esforço em prol da organização; e

c) um forte desejo de se manter como membro da organização. De acordo com Mowday et al. (1982), a noção de comprometimento afetivo está associada ainda à ideia de lealdade, sentimento de pertencer, desejo de contribuir e dar energia para a organização. Vale destacar que Mowday et al. (1982) recorreram ainda ao modelo proposto por Salancik (1977) para destacar a importância de características do trabalho que ampliem, no trabalhador, o "sentir-se responsável" por este. Tal sentimento de responsabilidade induziria a um envolvimento comportamental com o trabalho que, por sua vez, geraria atitudes consistentes.

O'Reilly e Chatman (1986), baseados na noção de processos de influência social, de Kelman (1958), distinguem três tipos de vínculo mantido entre trabalhador e organização: 
a) aquiescência, comportamento instrumental designado à obtenção de recompensas;

b) identificação, que ocorre quando os trabalhadores se comportam porque eles querem manter-se relacionados com a organização devido aos valores e metas que lhes são atrativos, embora esses valores e metas possam não ser pessoalmente adotados; e

c) internalização, que reflete o comportamento dirigido por valores e metas internos que sejam consistentes com os da organização.

Um exame empírico do modelo proposto por O’Reilly e Chatman (1986) demonstrou que resultados de correlação entre identificação e internalização tendem a formar padróes semelhantes, com elevada correlação positiva entre os fatores. Por outro lado, as correlaçôes entre esses dois fatores com aquiescência foram muito baixas ou negativas. Dado o nível de correlação entre identificação e internalização, há uma elevada probabilidade de que as dimensões venham a se referir a um mesmo construto e que o modelo de três fatores de Kelman não se sustente empiricamente para a caracterização do comprometimento organizacional.

Penley e Gould (1988), baseado no modelo tridimensional de Etzioni (1961), declararam que existem duas dimensões predominantes que caracterizariam o comprometimento com a organização: uma dimensão afetiva e uma instrumental. Enquanto a dimensão instrumental está relacionada a um sistema de compensações e recompensas recebidas por um indivíduo como um retorno do trabalho investido na organização, a dimensão afetiva está ligada ao apego emocional e senso pessoal de obrigação de cumprir com suas responsabilidades perante a organização. $\mathrm{O}$ comprometimento calculativo é assim tratado como o comprometimento instrumental. Já o comprometimento moral é considerado uma forma afetiva de comprometimento com a organização.

As pesquisas de Meyer e Allen constituem um marco importante na discussão sobre a dimensionalidade do construto comprometimento organizacional. Inicialmente, Meyer e Allen (1984) ressaltaram o caráter bidimensional do fenômeno, apontando as bases afetiva e de continuação como constituintes do comprometimento. Em 1990, estes autores adicionaram uma terceira dimensão: a normativa, fortemente influenciados pelo trabalho de Wiener (1982), que, por sua vez, foi derivado do modelo de intençôes comportamentais de Fishbein (1967). Allen e Meyer definem que a base normativa do compromisso está 
ligada aos sentimentos de obrigação dos trabalhadores de permanecerem na organização.

O modelo de Allen e Meyer (1990) traz contribuições significativas para o estudo da dimensionalidade do comprometimento organizacional, adicionando a noção de que a permanência em uma organização pode ser originária do desejo, da necessidade e, ou, do senso de dever ou obrigação. Estudos clássicos (Jaros, Jermier, Koehler \& Sincich, 1993; McGee \& Ford, 1987; Meyer \& Allen, 1991; Meyer, Allen \& Smith, 1993, 1997) afirmam a multidimensionalidade do construto, embora as pesquisas ainda não sejam conclusivas quanto aos seus componentes nem estabeleçam claramente seus antecedentes e consequentes (Bastos \& Brandão, 1993). Uma meta-análise conduzida pelo próprio J. Meyer et al. em 2002, aponta para uma possível sobreposição conceitual entre as dimensões afetiva e normativa, ao passo que a dimensão instrumental não se correlaciona com nenhuma daquelas dimensões (Meyer et al., 2002).

Outros estudos continuam a produzir resultados distintos sobre a dimensionalidade do comprometimento, como o de Delobbe e Vandenberghe (2000), que investigaram o comprometimento mediante o estudo de quatro dimensões (internalização, afetiva, de continuação e aquiescência) e o de Rego (2003), que hipotetiza um modelo hexadimensional (comprometimento afetivo, futuro comum, comprometimento normativo, sacrifícios avultados, escassez de alternativas e ausência psicológica).

Um importante estudo sobre a dimensionalidade do comprometimento organizacional foi conduzido por Solinger, Olffen e Roe (2008), que declararam a inconsistência do modelo tridimensional de Allen e Meyer (1990), destacando ainda que os três tipos de comprometimento não podem ser considerados como componentes do mesmo fenômeno atitudinal. Entre as inconsistências empíricas destacadas pelos autores, a primeira refere-se aos resultados de diferentes estudos, em que a base de continuação não se correlaciona ou se correlaciona negativamente com outros componentes (afetivo e normativo), assim como com outros correlatos atitudinais e variáveis relacionadas a trabalho, como cidadania e desempenho no trabalho (Cohen, 2003; Dunham, Grube \& Castañeda, 1994; Hackett, Bycio \& Hausdorf, 1994; Ko, Price \& Mueller, 1997; Meyer et al., 2002). Assim, o comprometimento atitudinal deveria ser composto unicamente pela base afetiva, o que tornaria o comprometimento organizacional um construto unidimensional. A Figura 2 apresenta alguns dos estudos de maior destaque sobre a dimensionalidade do comprometimento organizacional. 
Figura 2. Diferentes perspectivas acerca da dimensionalidade do comprometimento organizacional

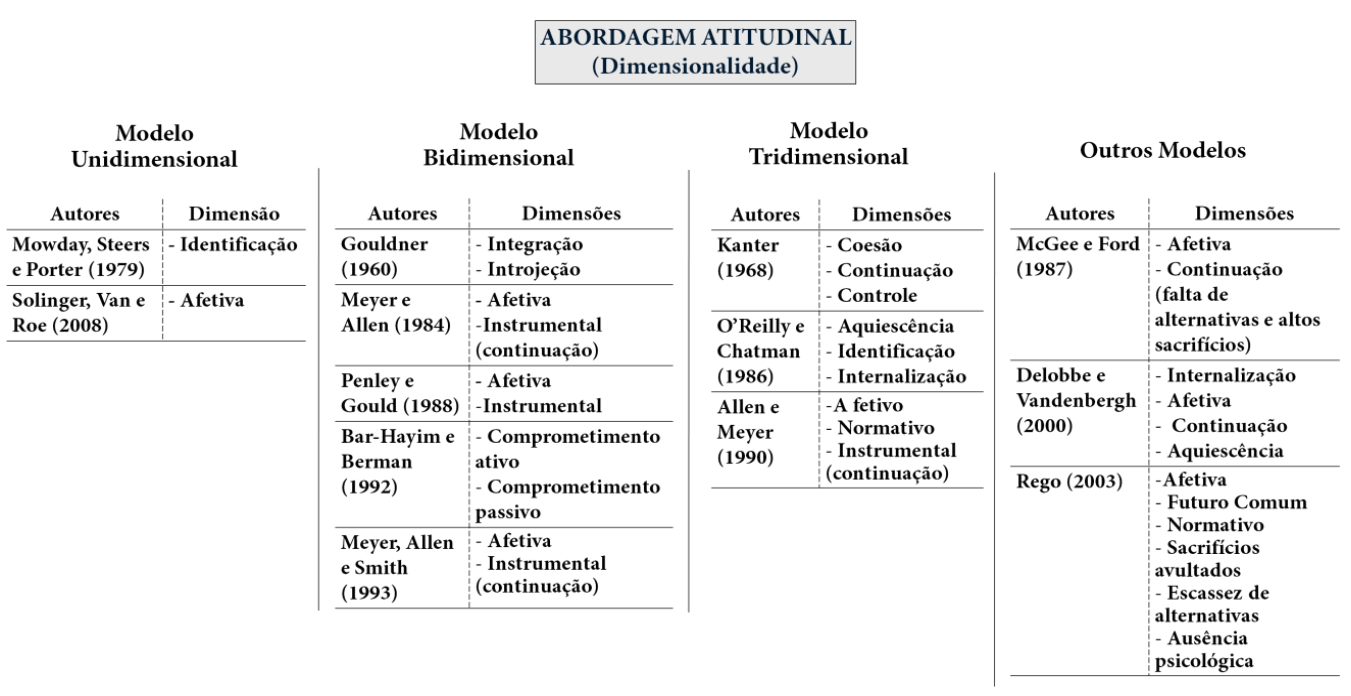

Fonte: elaborado pelos autores.

Nos últimos anos, a decisão de retomar a unidimensionalidade do comprometimento tem sido defendida em uma série de trabalhos brasileiros (Bastos \& Aguiar, 2015; Bastos, Maia, Rodrigues, Macambira \& BorgesAndrade, 2014; Rodrigues, 2009, 2011; Rodrigues \& Bastos, 2010; Silva, 2009, 2013). Para sustentar tal posicionamento, uma extensa agenda de pesquisa tem sido cumprida na tentativa de limitar o comprometimento organizacional àqueles elementos que constituem a sua essência, diferenciando-o de outros tipos de vínculos que parecem ter impactos muito distintos sobre o desempenho do trabalhador e da organização, como o entrincheiramento (Rodrigues, 2011; Rodrigues \& Bastos, 2010) e o consentimento organizacional (Silva, 2013; Silva $\&$ Bastos, 2010).

Além das fragilidades conceituais e empíricas que cercam o comprometimento organizacional, uma questão prática chama a atenção quando se pensa em sua dimensionalidade: na tentativa de proporcionar maior extensão e abrangência ao comprometimento organizacional, os modelos científicos propostos afastaram-se da forma como gestores e demais atores sociais compreendem esse vínculo. Tal distanciamento foi apontado por Bastos (1994) e pôde, mais recentemente, ser sistematicamente evidenciado por uma série de estudos que fizeram essa confrontação entre as concepções científicas e práticas do que é "ser comprometido" (Bastos, Brandão \& Pinho, 1997; Brito \& Bastos, 2001; Moscon, 2009; Moscon, Bastos \& Souza, 2012; Rowe \& Bastos, 2007). De um modo geral, tem-se que apenas os elementos diretamente relacionados à base afetiva são reconhecidos por gestores e funcionários como constituintes 
do comprometimento organizacional (Moscon et al., 2012). Esses resultados têm sido interpretados como importantes evidências adicionais de que a base afetiva do comprometimento deve, de fato, ser considerada como a essência desse vínculo do trabalhador com a sua organização.

\section{CONSIDERAÇÕES FINAIS}

Passados mais de 30 anos que Mowday et al. (1979) estabeleceram a primeira agenda de pesquisa sobre o comprometimento organizacional, muitas das questôes levantadas naquele momento não foram respondidas até hoje. $\mathrm{O}$ comprometimento organizacional continua sendo definido de variadas formas, centrado nos pressupostos da abordagem atitudinal e fortemente associado com outros construtos da área organizacional. Ainda são poucas as tentativas de revisão e integração do construto, como pode ser notado nos trabalhos clássicos de Becker (1960) e Reichers (1985), Bar-Hayim e Berman (1992), Bastos (1994), Mowday (1999), Meyer e Herscovitch (2001) e na meta-análise conduzida por Cooper-Hakim e Viswesvaran (2005), entre outros.

Embora este trabalho não tenha como objetivo propor uma nova agenda de pesquisa, sugere-se que trabalhos futuros focalizem alguns aspectos já sugeridos por outras agendas de pesquisa (Mathieu \& Zajac, 1990; Meyer, Allen \& Smith, 1997; Meyer et al., 2002; Mowday, 1999; Mowday et al. 1982), mas ainda não atendidos, bem como a inclusão de novos elementos de investigação. Entre os pontos já contemplados nas agendas de pesquisa, está a questão da dimensionalidade do comprometimento. Ao aceitar diferentes hipóteses acerca de quantas e quais seriam as dimensóes constitutivas do fenômeno, a literatura sobre comprometimento organizacional ainda mostra grande falta de consenso sobre sua definição.

Outro ponto crítico para uma agenda de pesquisa é o desequilíbrio entre as abordagens atitudinal e comportamental. O campo de pesquisas sobre o tema, ao preterir a investigação dos comportamentos que traduzam comprometimento organizacional, restringe a possibilidade da criação de um conceito mais integrador sobre o comprometimento. Assim, é premente que se considerem não somente crenças, sentimentos e preferências na investigação do fenômeno, mas, principalmente, a forma como atitudes e comportamentos se relacionam e se integram na caracterização do vínculo que o trabalhador estabelece com sua organização.

Contribuindo com essa dispersão conceitual, comumente o comprometimento organizacional tem sido descrito com base em outros construtos, como 
identificação, envolvimento, lealdade, cidadania, entre outros. Ainda são poucas as pesquisas que procuram testar a validade discriminante desse atributo diante de outros tipos de vínculos sociais estabelecidos entre trabalhador e organização. Assim, faz-se necessário que o construto seja caracterizado não somente em relação a aquilo que ele é, mas, fundamentalmente, que o distancie daquilo que ele não é.

Assim como fora sugerido por alguns autores (Bastos, 1993; Mathieu \& Zajac, 1990; Mowday et al., 1982), outro ponto da agenda que merece destaque diz respeito ao fato de que a pesquisa sobre comprometimento continua a carecer de uma maior quantidade de estudos longitudinais, que avaliem como o vínculo entre trabalhador e organização se forma e se mantém com o tempo. Estudos com esse recorte metodológico poderão fornecer elementos importantes para a própria definição do construto, ao avaliar a sua estabilidade ao longo do tempo.

De um modo geral, praticamente todos os pontos aqui sugeridos para uma agenda de pesquisa futura giram em torno da falta de clareza e precisão sobre a definição do comprometimento organizacional. Ora confundido com outros construtos, ora investigado praticamente por uma única abordagem teórica, a literatura sobre comprometimento organizacional ainda demanda estudos teóricos e empíricos que busquem propor e testar a interação entre diferentes variáveis atitudinais e comportamentais para a construção de uma definição operacional mais coesa sobre o fenômeno. 


\section{REFERÊNCIAS}

Achinstein, P. (1968). Concepts of science: a philosophical analysis. Baltimore: Johns Hopkins Press.

Allen, N. J. \& Meyer, J. P. (1990). The measurement and antecedents of affective, continuance, and normative commitment to the organization. Journal of Occupational Psychology, 63, 1-18.

Ashforth, B. E. \& Mael, F. (1989). Social identity theory and the organization. Academy of Management Review, 14, 20-39.

Bagozzi, R. P. \& Fornell, C. (1982). Theoretical concepts, measurements, and meaning. In C. Fornell (Org.). A second generation of multivariate analysis: measurement and evaluation. (pp. 24-38). New York: Praeger.

Bar-Hayim, A. \& Berman, G. S. (1992). The dimensions of organizational commitment. Journal of Organizational Behavior, 13 (4), 379-387.

Bastos, A. V. B. (1993). Comprometimento organizacional: um balanço dos resultados e desafios que cercam essa tradição de pesquisa. Revista de Administração de Empresas, 33 (3), 52-64.

Bastos, A. V. B. (1994). Comprometimento no trabalho: a estrutura dos vínculos do trabalhador com a organização, a carreira e o sindicato. (Tese de Doutorado). Escola de Administração, Universidade de Brasília, Brasília.

Bastos, A. V. B. \& Aguiar, C. V. N. (2015). Comprometimento organizacional. In K. Puente-Palácios \& A. L. Peixoto (Orgs.). Ferramentas de diagnóstico para organizaçôes e trabalho: um olhar a partir da psicologia. Porto Alegre: Artmed.

Bastos, A. V. B \& Brandão, M. G. A. (1993). Antecedentes de comprometimento organizacional em organizações públicas e privadas. In Associação Nacional de Pós-Graduação e Pesquisa em Administração (ANPAD) (Org.). Anais do 17 Encontro Anual da Associação Nacional de Pós-Graduação e Pesquisa em Administração (ANPAD). (pp. 102-116), Porto Alegre.

Bastos, A. V. B., Brandão, M. G. A. \& Pinho, A. P. M. (1997). Comprometimento organizacional: uma análise do conceito expresso por servidores universitários no cotidiano de trabalho. Revista de Administração Contemporânea, 1, 2, 97120.

Bastos, A. V. B., Maia, L. G., Rodrigues, A. C. A., Macambira, M. O. \& Borges- 
Andrade, J. E. (2014). Vínculos dos indivíduos com a organização: análise da produção científica brasileira 2000-2010. Psicologia: Teoria e Pesquisa, 30, 153-162.

Becker, H. S. (1960). Notes on the concept of commitment. American Journal of Sociology, 66(1), 32-42.

Benkhoff, B. (1997). Ignoring commitment is costly: new approaches establish the missing link between commitment and performance. Human Relations, 50(6), 701-726.

Beyer, J., Hannah, D. \& Milton, L. (2000). Ties that bind: Culture and attachments in organizations. In N. M. Ashkanasy, C. P. M. Wilderom \& M. F. Peterson (Eds.), Handbook of organizational culture \& climate. (pp. 323338). Thousand Oaks: Sage.

Bluedorn, A. C. (1982). A unified model of turnover from organizations. Human Relations, 35, 135-153.

Brito, A. P. M. P. \& Bastos, A. V. B. (2001). O schema de "trabalhador comprometido" e gestão do comprometimento: um estudo entre gestores de uma organização petroquímica. Organizaçōes e Sociedade, 8(22), 177-193.

Brown, M. E. (1969). Identification and some conditions of organizational involvement. Administrative Science Quarterly, 14, 346-355.

Cheney, G. (1982). Organizational identification as process and product: a field study. (Dissertação de Mestrado). Purdue University, West Lafayette.

Chen, Z. X., Tsui, A. S. \& Farh, J. L. (2002). Loyalty to supervisor vs. organizatinal commitment: relationships to employee performance in China. Journal of Occupational and Organizational Psychology, 75, 339-356.

Cohen, A. (2003). Multiple commitments in the workplace: an integrative approach. Mahwah: Lawrence Erlbaum Associates.

Cooper-Hakim, A. \& Viswesvaran, C. (2005). The construct of work commitment: testing an integrative framework. Psychological Bulletin, 131(2), 241-259.

Delobbe, N. \& Vandenberghe, C. (2000). A four-dimensional model of organizational commitment among belgian employees. European Journal of Psychological Assessment, 16(2), 125-138. 
Dunham, R. B., Grube, J. A. \& Castañeda, M. B. (1994). Organizational commitment: the utility of an integrative definition. Journal of Applied Psychology, 79, 370-380.

Etzioni, A. (1961). A comparative analysis of complex organizations. Glencoe: Free Press.

Fishbein, M. (1967). Attitude and the prediction of behavior. In M. Fishbein (ed.), Readings in attitude theory and measurement. (pp. 477-492). New York: Wiley.

Gouldner, H. P. (1960). Dimensions of organizational commitment. Administrative Science Quarterly, 4, 468-490.

Hackett, R. D., Bycio, P. \& Hausdorf, P. A. (1994). Further assessments of Meyer and Allen's three-component model of organizational commitment. Journal of Applied Psychology, 79, 15-23.

Hall, D. T. \& Schneider, B. (1972). Correlates of organizational identification as a function of career pattern and organizational type. Administrative Science Quarterly, 17(3), 340-350.

Jaros, S. J., Jermier, J. M., Koehler, J. W. \& Sincich, T. (1993). Effects of continuance, affective, and moral commitment on the withdrawal process: an evaluation of eight structural equation models. Academy of Management Journal, 36(5), 952-995.

Kanter, R. M. (1968). Commitment and social organization: a study and commitment mechanisms in utopian communities. American Sociological Review, 33, 499-517.

Katz, D. \& Kahn, R. L. (1974). Psicologia social das organizações. São Paulo: Atlas.

Kelman, H. C. (1958). Compliance, identification, and internalization: three processes of attitude change? Journal of Conflict Resolution, 2(1), 51-60.

Klein, H. J., Molloy, J. C. \& Cooper, J. T. (2012). Conceptual foundations: construct definitions and theoretical representations of workplace commitments. In H. J. Klein, T. E. Becker \& J. P. Meyer (Eds.). Commitment in organizations: accumulated wisdom and new directions (Vol. 1, pp. 3-36). New York: Routledge. 
Ko, J. W., Price, J. L. \& Mueller, C. M. (1997). Assessment of Meyer and Allen's three component model of organizational commitment in South Korea. Journal of Applied Psychology, 82, 961-973.

Lathan, G. P., Millman, Z. \& Karambaya, R. (1997). Content-domain confusion among researchers, managers, and union members regarding organizational citizenship behavior. Canadian Journal of Administrative Sciences, 14(2), 206212.

Lee, S. M. (1971). An empirical analysis of organizational identification. Academy of Management Journal, 14(2), 213-226

Lodahl, T. \& Kejner, M. (1965). The definition and measurement of job involvement. Journal of Applied Psychology, 49, 24-33.

Marsh, R. M \& Mannari, H. (1977). Organizational commitment and turnover: a predictive study. Administrative Science Quarterly, 22, 57-75.

Mathieu, J. E. \& Zajac, D. M. (1990). A review and meta-analysis of the antecedents, correlates, and consequences of organizational commitment. Psychological Bulletin, 108(2), 171-194.

McGee, G. W. \& Ford, R. C. (1987). Two (or more?) dimensions of organizational commitment: reexamination of the affective and commitment scales. Journal of Applied Psychology, 72, 638-642.

Menezes, I. G. (2006). Escalas de intençôes comportamentais de comprometimento organizacional (EICCO): concepção, desenvolvimento, validação e padronização. (Dissertação de Mestrado). Programa de Pós-Graduação em Psicologia, Universidade Federal da Bahia, Salvador.

Meyer, J. P. \& Allen, N. J. (1984). Testing the side-bets theory of organizational commitment: some methodological considerations. Journal of Applied Psychology, 69, 372-378.

Meyer, J. P. \& Allen, N. J. (1991). A three-component conceptualization of organizational commitment. Human Resource Management Review, 1, 61-89.

Meyer, J. P., Allen, N. J. \& Smith, C. A. (1993). Commitment to organizations and occupations: extension and test of a three-component conceptualization. Journal of Applied Psychology, 78(4), 538-551.

Meyer, J. P., Allen, N. J. \& Smith, C. A. (1997). Commitment in the workplace: 
theory, research and applications. Thousand Oaks: Sage.

Meyer, J. P. \& Herscovitch, L. (2001). Commitment in the workplace: toward a general model. Human Resource Management Review, 11, 299-326.

Meyer, J. P., Stanley, D. J., Herscovitch, L. \& Topolnytsky, L. (2002). Affective, continuance, and normative commitment to the organization: a meta-analysis of antecedents, correlates, and consequences. Journal of Vocational Behavior, $61,20-52$.

Mobley, W. H., Griffith, R., Hand, H. \& Megline, B. (1979). Review and conceptual analysis of the employee turnover process. Psychological Bulletin, $86,493-522$.

Morrow, P. (1983). Concept redundancy in organizational research: the case of work commitment. Academy of Management Review, 8, 486-500.

Moscon, D. C. B. (2009). Teorias implícitas de trabalhador comprometido $e$ estratégias cotidianas de gestão: uma análise qualitativa. (Dissertação de Mestrado). Universidade Federal da Bahia, Salvador.

Moscon, D. C. B., Bastos, A. V. B. \& Souza, J. A. J. (2012). É possível integrar, em um mesmo conceito, os vínculos afetivo e instrumental?: o olhar de gestores sobre o comprometimento com a organização. Organizações \& Sociedade, 19, 357-373.

Mowday, R. T. (1999). Reflections on the study and relevance of organizational commitment. Human Resource Management Review, 8(4), 387-401.

Mowday, R. T., Koberg, C. S. \& McArthur, A. W. (1984). The psychology of the withdrawal process: a cross-validation test of Mobley's intermediate linkages model of turnover in two samples. Academy of Management Journal, 27(1), 79-94.

Mowday, R. T., Porter, L. W. \& Steers, R. M. (1982). Employee-organization linkages: the Psychology of commitment, absenteeism, and turnover. New York: Academic Press.

Mowday, R. T., Steers, R. M \& Porter, L. W. (1979). The measurement of organizational commitment. Journal of Vocational Behavior, 14, 43-77.

O’Reilly, C. A. \& Caldwell, D. E. (1980). The commitment and job tenure of new employees: some evidence of postdecisional justification. Journal of 
Experimental Psychology, 16, 172-186.

O'Reilly, C. A. \& Chatman J. (1986). Organizational commitment and psychological attachment: the effects of compliance, identification, and internalization on prosocial behavior. Journal of Applied Psychology, 71, 492499.

Organ. D. W. \& Ryan, K. (1995). A meta-analytic review of attitudinal and dispositional predictors of organizational citizenship behavior. Personnel Psychology, 48, 775-802.

Osigweh, C. A. B. (1989). Concept fallibility in organizational science. Academy of Management Review, 16, 579-584.

Patchen, M. (1970). Participation, achievement, and involvement on the job. New Jersey: Prentice-Hall.

Penley, L. E. \& Gould, S. (1988). Etzioni’s model of organizational involvement: a perspective for understanding commitment to organizations. Journal of Organizational Behavior, 9, 43-59.

Peters, L. H., Bhagat, R. S. \& O'Connor, E. J. (1981). An examination of the independent and joint contributions of organizational commitment and job satisfaction on employee intentions to quit. Group and Organization Studies, $6,73-82$.

Porto, J. \& Tamayo, J. B. (2003). Desenvolvimento e validação da escala de civismo nas organizações. Estudos de Psicologia, 8(3), 393-402.

Rego, A. (2002). Climas de justiça nas organizações: sua relação com os comportamentos de cidadania. Caderno de Pesquisas em Administração, 9(1), $35-59$.

Rego, A. (2003). Comprometimento organizacional e ausência psicológica: afinal, quantas dimensões?. Revista de Administração de Empresas, 43(4), 25-35.

Reichers, A. E. (1985). A review and reconceptualization of organizational commitment. Academic Manager, 10(3), 465-76.

Riketta, M. (2005). Organizational identification: a meta-analysis. Journal of Vocational Behavior, 66, 358-384.

Robinson, S. L. \& Rousseau, D. M. (1994). Violating the psychological contract: 
not the exception but the norm. Journal of Organizational Behaviour, 15, 245259.

Rodrigues, A. C. A. (2009). Do comprometimento de continuação ao entrincheiramento organizacional: o percurso de validação da escala e análise da sobreposição entre os construtos. (Dissertação de Mestrado). Universidade Federal da Bahia, Salvador.

Rodrigues, A. C. A. (2011). Trabalhador entrincheirado ou comprometido? Delimitação dos vínculos do indivíduo com a organização. (Tese de Doutorado). Universidade Federal da Bahia, Salvador.

Rodrigues, A. C. A. \& Bastos, A. V. B. (2010). Problemas conceituais e empíricos na pesquisa sobre comprometimento organizacional: uma análise crítica do modelo tridimensional de J. Meyer e N. Allen. Revista Psicologia: Organizaçôes e Trabalho, 10, 129-144.

Rousseau, D. M. (1989). Psychological and implied contract in organizations. Employee Rights and Responsibilities Journal, 2, 121-139.

Rousseau, D. M. (1995). Psychological contract in organizations: understanding written and unwritten agreements. Thousand Oaks: Sage.

Rowe, D. E. O. \& Bastos, A. V. B. (2007). Comprometimento no trabalho: explorando o conceito, seus antecedentes e consequentes entre docentes universitários [CD]. In Anais do $1^{\circ}$ Encontro de Gestão de Pessoas e Relaçôes de Trabalho, Natal. 1 CD.

Salancik, G. R. (1977). Commitment and the control of organizational behavior and belief. In B. M. Shaw \& G. R. Salancik (orgs.), New directions in Organizational Behavior. (pp. 1-54). Chicago: St. Clair.

Salancik, G. R. \& Pfeffer, J. (1978). A social information processing approach to job attitudes and task design. Administrative Science Quarterly, 23, 224-253.

Silva, E. E. (2009). Trabalhador comprometido X obediente: explorando os limites conceituais e empiricos entre os construtos. (Dissertação de Mestrado). Universidade Federal da Bahia, Salvador.

Silva, E. E. (2013). Consentimento ou comprometimento?: delimitação conceitual e empírica dos vínculos com a organização. (Tese de Doutorado). Universidade Federal da Bahia, Salvador. 
Silva, E. E. \& Bastos, A. V. B. (2010). A escala de consentimento organizacional: construção e evidências de sua validade. Revista rPOT, 10(1), 7-22.

Solinger, O. N., Olffen, W. van \& Roe, R. A. (2008). Beyond the three-component model of organizational commitment. Journal of Applied Psychology, 93(1), 7083.

Steers, R. M. (1977). Antecedents and outcomes of organizational commitment. Administrative Science Quarterly, 22, 46-56.

Tajfel, H. (1978). Differentiation between social groups: studies in the social psychology of intergroup relations. London: Academic Press.

Ugboro, I. O. (1993). Loyalty, value congruency, and affective organizational commitment: an empirical study. Mid-America Journal of Business, 8(2), 2936 .

Wiener, Y. (1982). Commitment in organizations: a normative view. Academy of Management Review, 7(3), 418-428. 